\title{
Facilitators and barriers to participation in mental well-being programs by older Australians with vision impairment: community and stakeholder perspectives
}

\author{
Lisa Dillon $\mathbb{D}^{1,2} \cdot$ Diana Tang ${ }^{3} \cdot{\text { Gerald } \text { Liew }^{3} \cdot \text { Maree Hackett }}^{2,4} \cdot$ Ashley Craig ${ }^{5} \cdot$ Bamini Gopinath $\mathbb{1}^{3} \cdot$ Lisa Keay ${ }^{1,2}$
}

Received: 23 February 2020 / Revised: 17 May 2020 / Accepted: 19 May 2020 / Published online: 28 May 2020

(c) The Author(s), under exclusive licence to The Royal College of Ophthalmologists 2020

\begin{abstract}
Objective Older adults with vision impairment experience high rates of mental health problems, but very few access psychological support. We investigated community and stakeholder perspectives of the barriers and facilitators to participation in mental well-being programs for older adults with vision impairment.

Methods Adults aged $\geq 50$ years with vision impairment (community) were recruited from the client database, and low vision rehabilitation (LVR) professionals (stakeholders) from staff of a LVR provider. Participants completed one-on-one semi-structured interviews, which were designed and analyzed using behavior change theory.

Results Twenty-nine participants were interviewed; 16 community members and 13 stakeholders. Both groups cited mental health problems as a major concern, with many stakeholders reporting the grief and distress associated with vision loss experienced by their clients as having a negative impact on their mental and physical health. Major barriers to participation in mental well-being programs included a lack of awareness and difficulties accessing such programs, with stakeholders adding that their clients' lack of insight into their own mental health problems may reduce motivation to participate. Facilitators to participation in programs included the appeal of social interaction and inspirational speakers. An appropriate intervention could overcome these barriers, or enhance participation through education, persuasion, incentivisation, modeling, environmental restructuring, training, and enablement.

Conclusions While barriers were discussed more than facilitators to participation, there was general support for mental wellbeing programs. This study provides guidance from stakeholders for the development of mental well-being programs to address mental health problems in the growing number of older adults with vision impairment.
\end{abstract}

\section{Introduction}

Globally, it is estimated that the number of people living with vision impairment (encompassing low vision or

Supplementary information The online version of this article (https:// doi.org/10.1038/s41433-020-0992-z) contains supplementary material, which is available to authorized users.

Lisa Dillon

lisa.dillon1@unsw.edu.au

1 School of Optometry and Vision Science, Faculty of Science, UNSW Sydney, Sydney, NSW 2052, Australia

2 The George Institute for Global Health, Faculty of Medicine, UNSW Sydney, PO Box M201 Missenden Rd, Sydney, NSW 2050, Australia blindness that cannot be corrected with glasses or surgery) will rise from 38.5 million in 2020 to 115 million people by 2050; most of which (78\%) are aged 50 years or older [1]. Older age and vision impairment are associated with an increased risk of developing a mental health condition, particularly depression [2]. An estimated 69,519 nonindigenous Australians aged 50 years or above and 4282 indigenous Australians aged 40 years or above were living

3 Centre for Vision Research, Department of Ophthalmology, The Westmead Institute for Medical Research, The University of Sydney, 176 Hawkesbury Road Westmead, Sydney, NSW 2145, Australia

4 Faculty of Health and Wellbeing, University of Central Lancashire, Preston, Lancashire PR1 2HE, UK

5 John Walsh Centre for Rehabilitation Research, Northern Clinical School, Faculty of Medicine and Health, The University of Sydney, St. Leonards, Sydney, NSW 2650, Australia 
with irreversible vision impairment in 2016 [3]; all at consequent risk of mental health problems.

Older adults with vision impairment are approximately three times more likely to experience significant depressive symptoms, compared with those without vision impairment $[2,4]$. This is attributed to the impacts of vision impairment on functional capacity and activities of daily living [5-8]. Research suggests that low vision rehabilitation (LVR) services, and psychological interventions involving selfmanagement and problem-solving, may be effective in reducing depressive symptoms and depressive disorders among adults with vision impairment [9-12]. However, although $70 \%$ of adults with vision impairment report wanting psychological support, only $9 \%$ report receiving it [13], and it remains unclear why uptake of support is so low.

One study of LVR professionals' (hereafter stakeholders) perspectives of a mental well-being program found a number of barriers to the problem-solving treatment for primary care (PST-PC) being delivered in a LVR setting, that could be overcome through professional training, support, and improved screening [14]. The only other study reported on the reasons older adults with vision impairment withdrew early from PST-PC, and found responses ranging from the program being perceived as not relevant, to the program goals being achieved early [10]. Given this scant literature, the aim of this qualitative study is to investigate the barriers and facilitators to participation in a mental wellbeing programs, from the perspectives of communitydwelling older adults with vision impairment (community members) and stakeholders.

\section{Subjects and methods}

This qualitative study involved semi-structured, one-on-one telephone, or face-to-face interviews with community members and stakeholders, including orientation and mobility specialists and optometrists. This study was conducted within New South Wales (NSW) and the Australian Capital Territory (ACT), Australia.

Community members and stakeholders were recruited using purposive sampling through invitation letters and follow-up phone calls between August and October 2019. To address the study aim, we expected to recruit up to $20 \mathrm{com}$ munity members and up to 15 stakeholders for interview, or until data were saturated in each group, which was assessed by researchers (LD and $\mathrm{LK}$ ) at regular meetings. Community members were recruited from the client database of an Australian vision rehabilitation organization, Guide Dogs NSW/ ACT. Clients who had consented to be contacted regarding research were sent an e-mail and invited to contact the research team directly to express interest in the study. Participants were required to be aged 50 years and older and speak conversational English. Stakeholder participants were employed in a client-facing capacity by Guide Dogs NSW/ ACT, and were similarly sent an e-mail and invited to contact the research team directly to express interest in the study.

The behavior change wheel [15] was used as a framework to design the interview guides (Supplementary Files 1 and 2), and consequent analysis of the data. The behavior of interest was participation in a mental well-being program. Specifically, the wheel uses the COM-B model to explain an individual's propensity to adopt a behavior (see the inner wheel of Fig. 1). Behavior can be, in turn, targeted by nine intervention functions: coercion, education, enablement, environmental restructuring, incentivisation, modeling, persuasion, restriction, training [15]. The wheel also includes policy categories, which can impact the intervention functions; however, these were considered outside the scope of this study.

Semi-structured interviews, lasting 15-45 min, were conducted by two Master of Clinical Optometry students (AW and RM) and a Faculty of Medicine and Health $\mathrm{PhD}$ student (DT). The students were given initial training and ongoing support by two experienced public health qualitative researchers (LD and LK). Interviews were audio recorded using digital recorders, transcribed verbatim, and analyzed using NVivo software, using deductive analysis [16], following the COM-B and intervention functions of the behavior change wheel [15]. We took an iterative approach to data analysis [17], whereby data were revisited, coded, and themes discussed many times to ensure analytic reflexivity. Transcripts were coded separately by two of the students (AW and RM). Initial coding was then presented and critiqued in a meeting including all three students, chaired by LD. Two of the students (AW and RM) then collaborated to establish themes under each subset of the COM-B model, which were discussed and agreed upon at regular fortnightly meetings with LD and LK. Themes were then coded using intervention functions, and categorized as barriers or facilitators to participation in a mental well-being program by LD and DT.

\section{Patient and public involvement}

No patient under medical care or members of the general public were involved in the design, recruitment, or conduct of the study. However, there will be formal engagement with the LVR provider's advisory panel to discuss the next stages of developing and implementing a mental well-being program. Those participants who requested feedback will be informed via e-mail or telephone call about the results.

\section{Ethics approval}

Ethics approval was granted by the University of NSW Human Research Ethics Committee (HC190356). A 
Fig. 1 Barriers and facilitators to mental well-being program participation mapped on the behavior change wheel [15]. Barriers to participation appear in bold, while facilitators appear underlined, with their relative intervention function presented in the outer wheel.

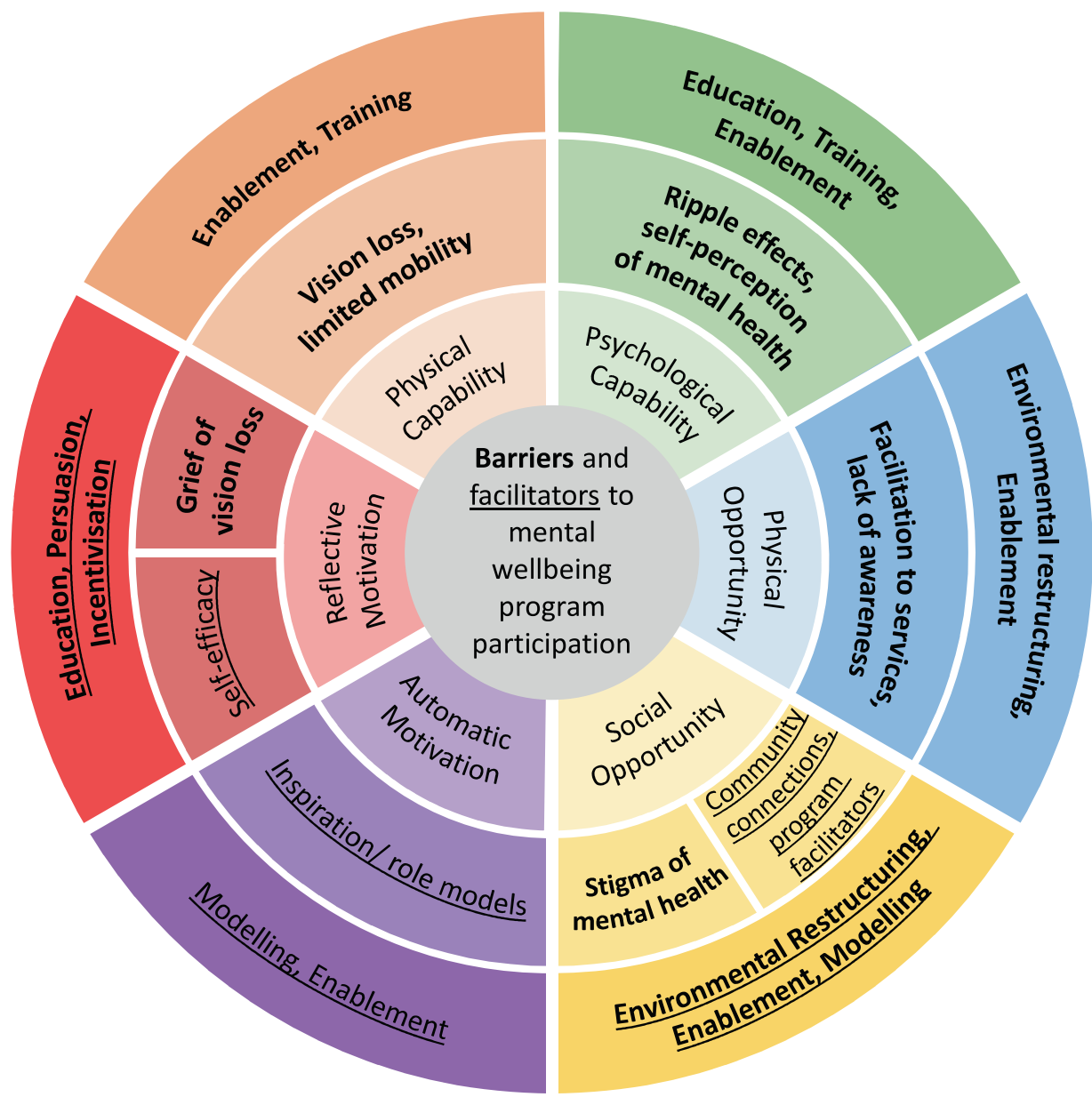

participation information statement was sent to interested participants, and oral informed consent was gained before commencement of the interview. The study is reported in line with the COREQ statement [18], supporting transparency in reporting of qualitative research.

\section{Results}

Of the 35 community members and stakeholders invited, 29 (83\%, 16 community members and 13 stakeholders) completed an interview. The 13 stakeholders included 12 orientation and mobility specialists and one optometrist. All stakeholder interviews were conducted over the phone, while seven of the community participants requested faceto-face interviews in their homes due to difficulties with hearing and/or accessing a phone.

We identified 12 themes (Fig. 1); eight represented barriers, and four facilitators to mental well-being program participation. Illustrative quotes associated with each theme are presented below, with intervention functions in brackets next to the COM-B component. Participants are identified as $\mathrm{C}$ for community members and $\mathrm{S}$ for stakeholders, followed by an identification number.

Both participant groups showed a high level of interest through long and engaging discussions around the mental well-being of people with vision impairment. Stakeholders expressed that many of their clients have concerns regarding their mental well-being, indicating the importance of this topic and need for such programs:

"I'm finding most of the clients that I...work with, express that they have anxiety, depression, or have had nervous breakdowns, or are currently having mental health issues." (S002)

\section{Barriers}

\section{Physical capability (Enablement and Training)}

Mobility impacts participation Participants reported difficulties in mobility as a result of their vision loss, which 
prevented them from engaging with their community and/or programs: "Because you can't go out. That's the biggest
problem..." $(\mathrm{C} 008)$

“So, you don't tread on little kids or get tangled up in dog leads...that's always in the back of your mind. I think I'll just stay at home." (C004)

In particular, there were concerns regarding loss of the ability to drive:

"You're not going to get them in a group scenario, but also they can't drive so they can't get to anywhere." (S008)

Some participants also found that their mobility limitations and location of residence interfered with their ability to connect with other individuals with vision impairment and contributed to feelings of isolation:

"I would love to have a group of other people who were also vision impaired, but they seem to be scattered all over the country." (C020)

Vision loss impacts everyday activities The inability to accomplish activities of daily living deterred participation in programs and other activities:

"Because of their vision impairment, I don't think they integrate as much with others in their community." (S009)

"I can't watch TV and I do like TV actually. I can't read anything anymore and I used to love a newspaper." (C020)

\section{Psychological capability (Education, Training, and Enablement)}

Ripple effects Stakeholders expressed concerns about mental health problems extending into other aspects of their client's lives and preventing participation in mental wellbeing programs:

"Stress and mental health, the physiological changes to the body... impacts on people's functional vision.
There's all these ripple effects if the mental health component is not addressed." (S006)

"I'd be the only person they'd see that week and maybe they'd have a cry...need to work through the issues they have before they can start doing routes and getting out in their community." (S015)

Self-perception of mental health The acknowledgment of having a mental health problem varied among participants:

"One very good friend who's also legally blind... when I bring up the subject with him he says, Oh, better not even to think about it. And he's not really getting all the services that he should be getting as a blind person." (C004)

"Has it affected me? I lost my licence. I'm pretty much housebound. My doctor wants me to see... a psychologist. And I said, No, I'm not that bad.” (C008)

"I try to prepare myself for the future, but I don't think it's affected my mental health, as far as others are concerned anyway." (C009)

\section{Physical opportunity (Environmental Restructuring and Enablement)}

Facilitation of services Participants expressed concerns about their ability to access mental well-being programs following their vision loss:

"When I lost my sight I had to really scrabble and... call for information." (C007)

"If there is support, they [support provider] could call our clients ...[so] the client doesn't have to initiate [seeking services] themselves...because I have a lot of clients who once they lose their vision, they're not able to navigate a phone." (S003)

Stakeholders also expressed their concerns about how they can best assist their clients to receive support for their mental health problems: 
"If we had a script or a proper format that once a client has identified that they've got mental health issues that we should say because you've mentioned that to us can we write some information down and pass it on to someone to suggest help or at least to refer." (S013)

Lack of awareness of services Community and stakeholders were unaware of current mental well-being programs targeted at Australians with vision impairment:

"I'm not aware of any program for... people with vision impairment." (S012)

"In a major Sydney hospital and they didn't ever say to me that I needed to be referred to Vision Australia or Guide Dogs or anywhere like that. And I just said, Okay, well, I can do this. But the trouble is I couldn't see." (C005)

\section{Social opportunity (Environmental restructuring, Enablement, and Modeling)}

Stigma about mental health problems Participants described the negative stigma surrounding mental health issues as a barrier to participation:

“...it doesn't seem to be talked about a lot.” (C015)

"People have a perception, there's still a stigma around mental illness, so a lot of them might not disclose." (S005)

"I would say don't call it a mental health program.” (C005)

\section{Reflective motivation (Education, Persuasion, and Incentivisation)}

Grief associated with vision loss Feelings of grief and beliefs regarding vision loss can make participation and engagement unmotivating:

"If a person is depressed and feels like they can't do anything, then it's likely they're not going to go into orientation or mobility training with high expectations, and a good learning frame of mind." (S012)

\section{Facilitators}

\section{Social opportunity (Environmental restructuring,} Enablement, and Modeling)

Community connections The desire for a connection to the community particularly with those experiencing similar vision loss was described by participants:

“...having a group that comes together that has a focus on adapting to change and understanding... I think is fairly powerful." (C015)

"Although technology and online stuff is cool and innovative...it sort of takes away from that human connection that you have when you sit in the same room as somebody." (S015)

"There's others there to talk to and pass the time of day with. It's not a discussion group, it's just to pass the time. You know, with the social aspect of it, but they're getting to know me." (C003)

"For so long I just thought our family was a family of freaks because not knowing of anyone else that had it... 30 years ago I joined up with the Nepean Blind Sports Club...I met a couple of people with the same condition and... went to social events out there." (C012)

Who can facilitate mental well-being programs Participants also expressed their opinions about who they think would be best suited to deliver low vision mental well-being programs. Different levels of expertise were articulated ranging from lay-facilitators to mental health professionals like psychologists:

"Anybody who has got group-based skills and some level of working with groups." (C005)

"I always feel that if someone's been through a situation they're the ones I think are the better ones." (C012) 
A consultant psychologist with a good knowledge of grief... it would be great to be able to run scenarios by that person. And say, this is what I'm dealing with, or this is what I've done. It would be lovely to be able to say to clients If you want to talk more about this, we have a psychologist on staff that might be able to give you a ring." (S004)

"It would be good if we had one person that they could make a call to that has more specific information and then can put them in touch with people in their own area... or what organisations are close to them." (S013)

\section{Automatic motivation (Modeling and Enablement)}

Inspiration/role model Some participants stated how a role model is an inspiring motivation for participating in mental well-being programs:

"Those have been some of our better meetings when we've been inspired by others in other words." (C009)

“...hearing people's stories about how they might have done it is really powerful for people who might be going through that part of the process." (C005)

\section{Reflective motivation (Education, Persuasion, and Incentivisation)}

Self-efficacy Many participants believed they were selfsufficient and capable of handling their own issues:

"I can't change it, I've just got to learn to adjust to it and that's just going to be my life." (C011)

"I don't feel sorry for myself, there's no point. I've got two legs, I can walk, so life's good." (C022)

\section{Discussion}

Consistent with evidence of the high burden of mental health problems in older adults with vision impairment $[2,4]$, we found strong interest in developing mental wellbeing programs among community members and stakeholders. The interviews revealed that both groups acknowledged the substantial negative impacts of mental health problems. However, both groups were unaware of any mental well-being programs specific to people with vision impairment, thus, confirming the importance of developing a mental well-being program tailored to this population group.

We identified two times more barriers than facilitators (i.e., eight vs. four) to effective participation in a mental well-being program. The intervention function Enablement was relevant to all three facilitators, and the majority of barriers. Enablement refers to increasing means, and reducing barriers [15], and is key to designing a program for this population. A major concern related to Enablement, along with Environmental Restructuring (changing the physical or social context [15]), was functional disability. Many community members felt that vision loss limited their capability and opportunity to access programs due to difficulty in reading or finding information, and traveling to where programs were held. Travel concerns are a common barrier in this population [19], and are influenced by the severity of vision impairment, distance to program location, and availability of support people. Recommendations to overcome these barriers, as they relate to intervention functions [15], include: producing material in larger, easy-to-read print (Enablement); LVR professionals telling community members what options are available (Education; increasing knowledge or understanding); and program organizers making community transport available (Environmental Restructuring) or provide orientation and mobility services (Training; imparting skills), if travel is required.

In addition to physical limitations, the acknowledgment of having a mental health problem influenced participation and varied among participants. Some mentioning conditions like depression and anxiety, others downplaying any concerns and associating it with complaining, and the remainder stating that their vision loss did not have any impact on their mental health. The intervention functions of Modeling (an example for people to aspire to or imitate) and Education are particularly relevant to alleviate these concerns and key to tackling the barriers of social stigma and selfperception. Nyman et al. [20] identified that vision impairment can impact psychosocial well-being through social isolation; while acceptance of vision loss and social support were facilitators of adjustment. We also identified self-efficacy as a facilitator, which has been shown to lead to good mental health outcomes in other contexts [21]. Given there was general consensus among both groups of the benefits of social connection and positive role models, it is recommended interventions include this in their design.

A pilot feasibility study in Australia recently investigated the delivery of PST-PC by LVR professionals to older adults with vision impairment who met the criteria for mild 
symptoms of depression [10]. PST-PC, based on cognitive behavioral therapy principles, is a low-intensity approach designed to assist with functional adjustment, resilience building, and generalized well-being. PST-PC can be delivered by nonmental health professionals, face-to-face, as well as over the phone, or over video conference, significantly increasing its reach. Holloway et al. [10] found that those that who stayed in the study had significant reductions in depressive symptoms, and improvements in health-related quality-of-life and problem-focused coping. Those who withdrew from the study early were typically older, with complex needs, as has been found in other studies [22-24]. Thus, it is critical that any future interventions must consider methods to retain adherence of older participants with competing health priorities.

Varying levels of mental health problems and requirements for support were reported by community members as well as stakeholders about their clients. A steppedcare approach may be the most efficient in this context, as not all clients require the same type or intensity of intervention [25]. Stepped-care comprises different intervention components, with the idea that if the first, less intensive step does not lead to a reduction in symptoms, then a person moves to the next step, consisting of more intensive and potentially more expensive interventions [9]. Several randomized controlled trials conducted outside the field of low vision have found that a stepped-care approach can be effective in minimizing depression and/or anxiety $[26,27]$, and has been endorsed to address depression in older adults in clinical guidelines, such as the UK NICE guidelines [28]. The ideas for interventions identified in this study could be integrated within a stepped-care approach, including social groups, psychologist referral, and PST-PC.

\section{Strengths and limitations}

This is the first study to explore the perspectives of older adults with vision impairment and client-facing professionals involved in LVR regarding mental health problems. The inclusion of client-facing professionals provides essential insight needed to design a stakeholder-driven intervention. A second strength is the semi-structured nature of the interviews, which allowed for tailored discussions to explore each participant's personal outlook and interpretation of the impacts of mental health problems and vision impairment. However, this also resulted in interviews of varying length, and occasional tangents in conversation. Despite compelling findings, the authors acknowledge that the study is limited to one community organization and therefore the presented results are only a preliminary indicator of the mental health perspectives in this population group. Moreover, community member participants were recruited through purposive sampling from the client base of one Australian LVR provider, and specifically only those clients who had agreed to be contacted about participating in research, and had also agreed to participate in this particular study. This potential selection bias may have influenced our results, and may limit the generalizability of findings to those not associated with an Australian LVR provider, or those not interested in participating in research or this particular study. Similarly, stakeholder participants were orientation and mobility specialists and one optometrist from the one Australian LVR organization, which may limit the generalizability of findings to these LVR providers in other organizations, or other professions involved in LVR, such as occupational therapists, orthoptists, assistive technology specialists, and social workers. Nonetheless, as data saturation was achieved, we are confident the range of themes and corresponding intervention functions suitably address the study aim. Furthermore, information on visual acuity, cause of vision loss, and presence of comorbidities was not available for collection. This information may have been valuable in terms of interpreting the qualitative data.

This study has several key implications for clinical practice. First, improving the mental well-being of visually impaired older adults is a priority. We postulate this may enhance their participation in other programs, such as orientation and mobility, and this, in turn, could improve their physical health, community connections, and overall well-being. Second, LVR professionals, such as orientation and mobility specialists and optometrists, are in a unique position to identify those at risk of, or already experiencing mental health problems and can refer as necessary, if provided with the necessary training. Third, older adults with vision impairment may benefit from appropriate education and training to better support them as they adjust to living with a vision impairment. We suggest this may be in the form of evidence-based education regarding their vision diagnosis and prognosis, or psychological support from an appropriately trained mental health practitioner. Fourth, providing older adults with vision impairment the opportunity to connect with others in similar situations, as well as those who are living well with vision impairment, is likely to improve their community connections and social engagement. As a next step, the suggestions for future interventions collected here will be presented through round tables with older adults with vision impairment and service providers. This will ensure that any developed strategies are practical and acceptable to the community and stakeholders, with the aim to improve the ultimate adoption and scalability of a mental well-being program. 


\section{Summary}

\section{What was known before}

- Older adults with vision impairment experience high rates of mental health problems.

- Although the majority of older adults want psychological support, very few receive it.

\section{What this study adds}

- Both older adults with vision impairment, and the professionals who work with them consider mental health problems as a major concern.

- Facilitators to participation in mental health programs include community connection and inspirational speakers.

- Barriers to participation include lack of awareness of programs, physical limitations, and self-perception of mental health.

Acknowledgements We would like to acknowledge students Rachel Muyco and Aimee Wassermann for conducting many of the semistructured interviews along with DT. We would also like to acknowledge Kerrie Ren for her assistance with study administration, as well as Guide Dogs NSW/ACT.

Funding This research was funded by the UNSW Sydney-University of Sydney: Mental Health and Wellbeing-Early Intervention and Prevention (Older People) Seed Funding Scheme.

\section{Compliance with ethical standards}

Conflict of interest The authors declare that they have no conflict of interest.

Publisher's note Springer Nature remains neutral with regard to jurisdictional claims in published maps and institutional affiliations.

\section{References}

1. Bourne RRA, Flaxman SR, Braithwaite T, Cicinelli MV, Das A, Jonas JB, et al. Magnitude, temporal trends, and projections of the global prevalence of blindness and distance and near vision impairment: a systematic review and meta-analysis. Lancet Glob Health. 2017;5:e888-97.

2. Court H, McLean G, Guthrie B, Mercer SW, Smith DJ. Visual impairment is associated with physical and mental comorbidities in older adults: a cross-sectional study. BMC Med. 2014;12:181.

3. Foreman J, Keel S, Xie J, cvan Wijngaarden P, Crowston J, Taylor HR, et al. National Eye Health Survey. 2016. https://www. vision2020australia.org.au/resources/national-eye-health-surveyreport/.

4. Evans JR, Fletcher AE, Wormald RP. Depression and anxiety in visually impaired older people. Ophthalmology. 2007;114:283-8.
5. Nyman SR. Psychosocial issues in engaging older people with physical activity interventions for the prevention of falls. Can J Aging. 2011;30:45-55.

6. Brody BL, Gamst AC, Williams RA, Smith AR, Lau PW, Dolnak $\mathrm{D}$, et al. Depression, visual acuity, comorbidity, and disability associated with age-related macular degeneration. Ophthalmology. 2001;108:1893-900.

7. Rovner BW, Casten RJ, Hegel MT, Massof RW, Leiby BE, Ho $\mathrm{AC}$, et al. Improving function in age-related macular degeneration: a randomized clinical trial. Ophthalmology. 2013;120:1649-55.

8. Casten R, Rovner BW, Leiby BE, Tasman W. Depression despite anti-vascular endothelial growth factor treatment of age-related macular degeneration. Arch Ophthalmol. 2010;128:506-8.

9. van der Aa HP, van Rens GH, Comijs HC, Bosmans JE, Margrain $\mathrm{TH}$, van Nispen RM. Stepped-care to prevent depression and anxiety in visually impaired older adults-design of a randomised controlled trial. BMC Psychiatry. 2013;13:209.

10. Holloway E, Sturrock B, Lamoureux E, Hegel M, Casten R, Mellor D, et al. Delivering problem-solving treatment in lowvision rehabilitation: a pilot feasibility study. Rehabil Psychol. 2018;63:349-56.

11. van der Aa HP, Margrain TH, van Rens GH, Heymans MW, van Nispen RM. Psychosocial interventions to improve mental health in adults with vision impairment: systematic review and metaanalysis. Ophthalmic Physiol Opt. 2016;36:584-606.

12. Kamga H, McCusker J, Yaffe M, Sewitch M, Sussman T, Strumpf E, et al. Self-care tools to treat depressive symptoms in patients with age-related eye disease: a randomized controlled clinical trial. Clin Exp Ophthalmol. 2017;45:371-8.

13. Sturrock B. Treating depression in people with vision impairment. 2018. https://www.psychology.org.au/for-members/publications/ inpsych/2018/feb/Treating-depression-in-people-with-vision-impa irm.

14. Holloway E, Sturrock B, Lamoureux E, Keeffe J, Hegel M, Casten $\mathrm{R}$, et al. Can we address depression in vision rehabilitation settings? Professionals' perspectives on the barriers to integrating problem-solving treatment. Disabil Rehabil. 2018;40:287-95.

15. Michie S, van Stralen MM, West R. The behaviour change wheel: a new method for characterising and designing behaviour change interventions. Implement Sci. 2011;6:42.

16. Vaismoradi M, Turunen H, Bondas T. Content analysis and thematic analysis: implications for conducting a qualitative descriptive study. Nurs Health Sci. 2013;15:398-405.

17. Srivastava P, Hopwood N. A practical iterative framework for qualitative data analysis. Int J Qualitative Methods. 2009;8:76-84.

18. Tong A, Sainsbury P, Craig J. Consolidated criteria for reporting qualitative research (COREQ): a 32-item checklist for interviews and focus groups. Int J Qual Health Care. 2007;19:349-57.

19. Dillon L, Duffy P, Tiedemann A, Keay L. Acceptability of fall prevention strategies for older people with vision impairment. Int J Orientat Mobil. 2018;9:1-9.

20. Nyman SR, Dibb B, Victor CR, Gosney MA. Emotional wellbeing and adjustment to vision loss in later life: a meta-synthesis of qualitative studies. Disabil Rehabil. 2012;34:971-81.

21. Craig A, Nicholson Perry K, Guest R, Tran Y, Middleton J. Adjustment following chronic spinal cord injury: determining factors that contribute to social participation. Br J Health Psychol. 2015;20:807-23.

22. Nollett CL, Bray N, Bunce C, Casten RJ, Edwards RT, Hegel MT, et al. High prevalence of untreated depression in patients accessing low-vision services. Ophthalmology. 2016;123:440-1.

23. Boeschoten RE, Dekker J, Uitdehaag BM, Polman CH, Collette $\mathrm{EH}$, Cuijpers $\mathrm{P}$, et al. Internet-based self-help treatment for 
depression in multiple sclerosis: study protocol of a randomized controlled trial. BMC Psychiatry. 2012;12:137.

24. Reinhardt JP, Horowitz A, Cimarolli VR, Eimicke JP, Teresi JA. Addressing depression in a long-term care setting: a phase II pilot of problem-solving treatment. Clin Ther. 2014;36:1531-7.

25. Haaga DA. Introduction to the special section on stepped care models in psychotherapy. J Consult Clin Psychol. 2000;68:547-8.

26. Patel V, Weiss HA, Chowdhary N, Naik S, Pednekar S, Chatterjee $\mathrm{S}$, et al. Effectiveness of an intervention led by lay health counsellors for depressive and anxiety disorders in primary care in
Goa, India (MANAS): a cluster randomised controlled trial. Lancet. 2010;376:2086-95.

27. van't Veer-Tazelaar PJ, van Marwijk HW, van Oppen P, van Hout HP, van der Horst HE, Cuijpers P, et al. Stepped-care prevention of anxiety and depression in late life: a randomized controlled trial. Arch Gen Psychiatry. 2009;66:297-304.

28. National Institute for Health and Care Excellent (NICE). Common mental health problems: identification and pathways to care. Clinical guideline [CG123]. 2001. https://www.nice.org.uk/guida nce/CG123/chapter/1-Guidance\#stepped-care. 